\title{
Transient Symptomatic Downregulation of Cortical Neurotransmitter Receptor Function Due to Cerebral Hyperperfusion after Arterial Bypass Surgery for a Patient with Ischemic Moyamoya Disease
}

\author{
Yasuyoshi SHIMAdA, ${ }^{1}$ Daigo KoJIMA, ${ }^{1}$ Jun YoshIDA, ${ }^{1}$ Masakazu KOBAYASHI, ${ }^{1}$ \\ Kenji YOSHIDA, ${ }^{1}$ Shunrou FUjIWARA, ${ }^{1}$ and Kuniaki OGASAWARA ${ }^{1}$
}

${ }^{1}$ Department of Neurosurgery, Iwate Medical University School of Medicine, Morioka, Iwate, Japan

\begin{abstract}
Cerebral hyperperfusion syndrome following arterial bypass surgery is known as a surgical complication of moyamoya disease (MMD). How cerebral hyperperfusion affects neural function and causes neurological deficits remains unknown. We report here a case with cerebral hyperperfusion syndrome after arterial bypass surgery for ischemic MMD. Chronological changes of brain perfusion and central benzodiazepine receptor biding potential were observed using single-photon emission computed tomography. A 20-year-old woman with ischemic MMD underwent arterial bypass surgery. Six days later, cerebral hyperperfusion syndrome developed. During this syndrome, contralateral-to-ipsilateral cerebellar asymmetry of blood flow and a decrease in central benzodiazepine receptor binding potential in the area with hyperperfusion were observed. Four months later, these two findings resolved and a neurological examination revealed no abnormal signs. Cerebral hyperperfusion after arterial bypass surgery for ischemic MMD may lead to transient, reversible reduction of cerebral metabolism and downregulation of cortical neurotransmitter receptor function, resulting in transient neurological deficits.
\end{abstract}

Key words: moyamoya disease, arterial bypass surgery, hyperperfusion, neurotransmitter receptor, downregulation

\section{Introduction}

Moyamoya disease (MMD) is a chronic, occlusive cerebrovascular disease of unknown etiology characterized by bilateral steno-occlusive changes in the terminal portion of the internal carotid artery (ICA) and an abnormal vascular network at the base of the brain. ${ }^{1,2)}$ Revascularization surgery, such as superficial temporal artery (STA)-middle cerebral artery (MCA) anastomosis, is generally employed as the standard surgical treatment for MMD with the onset of ischemic symptoms. ${ }^{3)}$ Cerebral hyperperfusion following arterial bypass surgery is known as a surgical complication of MMD and is defined as an acute substantial increase in ipsilateral cerebral blood flow (CBF) well

Received June 4, 2018; Accepted August 20, 2018

Copyright $\odot 2018$ by The Japan Neurosurgical Society This work is licensed under a Creative Commons AttributionNonCommercial-NoDerivatives International License. above the metabolic demands of the brain tissue. ${ }^{4-6)}$ Cerebral hyperperfusion syndrome is a complication of cerebral hyperperfusion, with an incidence of approximately $30 \%$ and with characteristic features including transient aphasia, hemiparesis, and dysarthria. ${ }^{4,7,8)}$ However, how cerebral hyperperfusion affects neural function and causes neurological deficits remains unknown. Brain perfusion can be assessed using single-photon emission computed tomography (SPECT) with ${ }^{123} \mathrm{I}-\mathrm{N}$-isopropyl-p-iodoamphetamine $\left({ }^{123} \mathrm{I} \text {-IMP }\right)^{9)}$. Central benzodiazepine receptors are widely and abundantly expressed in the cerebral cortex. Their distribution in the human brain can be visualized with SPECT using ${ }^{123}$ I-iomazenil, which binds to these receptors. ${ }^{10)}$

Here, we present a case involving a patient with cerebral hyperperfusion syndrome after arterial bypass surgery for ischemic MMD. The patient underwent brain perfusion and neurotransmitter receptor biding potential imaging using SPECT before surgery, during 
hyperperfusion syndrome, and after resolution of this syndrome.

\section{Case Presentation}

A 20-year-old woman with a history of juvenile hypertension presented with recurrent transient ischemic attacks manifesting as right motor weakness during hyperventilation. At visiting our department, a neurological examination revealed no abnormal signs. Magnetic resonance imaging (MRI) of the head revealed bilateral steno-occlusive changes in the terminal portion of the ICA and multiple small signal voids in the bilateral basal ganglia without ischemic lesions in the whole brain. Cerebral angiography through arterial catheterization showed MMD.

The patient underwent a single STA-MCA anastomosis with indirect revascularization including encephalo-myosynangiosis and encephalo-duroarterio-synangiosis in the left fronto-temporal region. The M4 of the left MCA, that perfused the pre-motor area, was selected as a recipient artery. The postoperative course was uneventful until 6 days after surgery when motor aphasia and right hemiparesis newly developed. MRI revealed no ischemic cerebral lesions and a patent bypass (Fig. 1). Systolic arterial blood pressure was intensively controlled between 100 and $140 \mathrm{mmHg}$ by intravenous antihypertensive drugs. The symptoms lasted for 10 days, and after that, gradually resolved. On the 4 th postoperative month, a neurological examination revealed no abnormal signs and no further neurological deficits developed. MRI demonstrated no ischemic cerebral lesions.

The institutional ethics committee at our institution reviewed and approved the below study protocol. The patient provided written informed consent

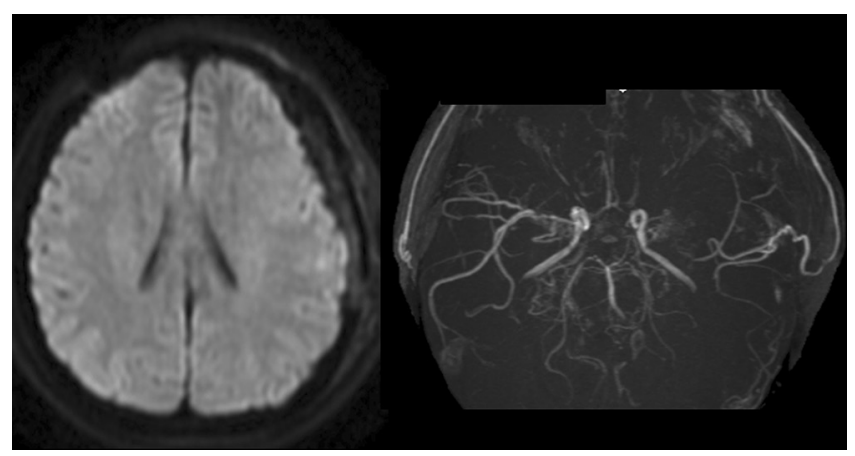

Fig. 1 Diffusion-weighted magnetic resonance image at the day when motor aphasia and right hemiparesis newly developed shows no ischemic cerebral lesions (left). Magnetic resonance angiography performed simultaneously demonstrates a patent superficial temporal artery-middle cerebral artery anastomosis (right). prior to participation. Brain perfusion (Fig. 2) and central benzodiazepine receptor biding potential (Fig. 3) imaging were performed using SPECT with ${ }^{123}$ I-IMP and ${ }^{123}$ I-iomazenil, respectively, in the same manner described previously., ${ }^{9,10)}$ The former was performed 7 days before surgery, 8 days after surgery ( 2 days after development of postoperative

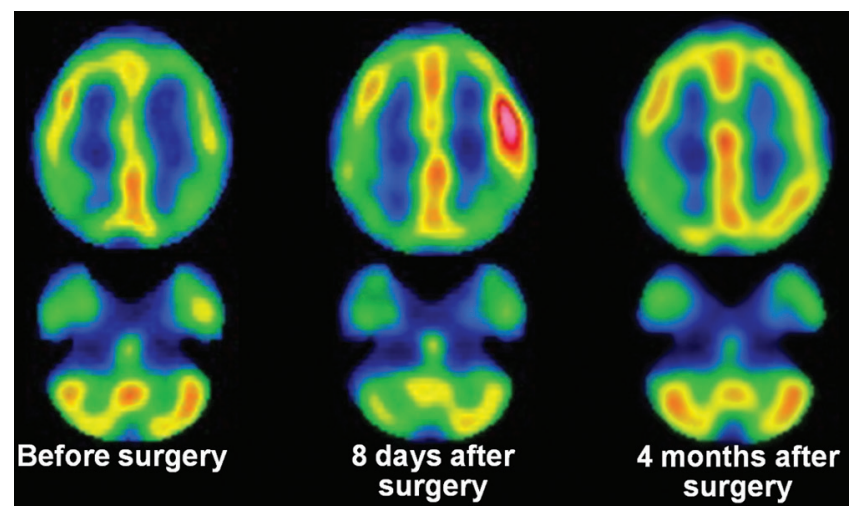

Fig. 2 Brain perfusion single-photon emission computed tomography (SPECT) images with ${ }^{123} \mathrm{I}-\mathrm{N}$-isopropyl-piodoamphetamine. At 7 days before surgery, blood flow is lower in the left cerebral hemisphere than in the right cerebral hemisphere (left). New neurological deficits, such as motor aphasia and right hemiparesis developed 6 days after surgery. During development of these deficits ( 2 days after the onset), blood flow is prominently increased in the left pre-motor area and blood flow in the right cerebellar hemisphere is decreased relative to blood flow in the left cerebellar hemisphere (middle). On the 4th postoperative month when a neurological examination revealed no abnormal signs, increased blood flow in the left pre-motor area and contralateral-to-ipsilateral cerebellar asymmetry of blood flow disappear (right).

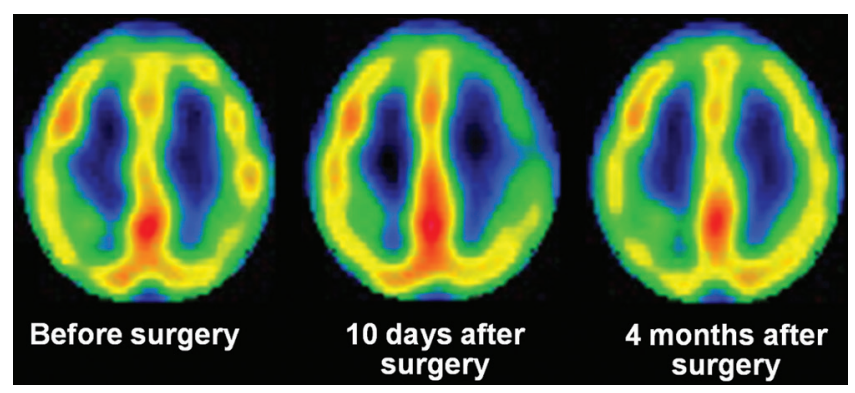

Fig. 3 Central benzodiazepine receptor biding potential SPECT images with ${ }^{123}$ I-iomazenil. At 5 days before surgery, central benzodiazepine receptor biding potential is slightly lower in the left cerebral hemisphere than in the right cerebral hemisphere (left). During development of new postoperative neurological deficits (4 days after the onset), central benzodiazepine receptor biding potential is prominently decreased in the left frontal lobe (middle). On the 4 th postoperative month, the decrease in central benzodiazepine receptor biding potential in the left frontal lobe disappears (right). 
neurological deficits), and 4 months after surgery; the latter was performed 2 days after each brain perfusion SPECT imaging.

Preoperatively, CBF was lower in the left cerebral hemisphere than in the right cerebral hemisphere. Central benzodiazepine receptor biding potential was slightly lower in the left cerebral hemisphere than in the right cerebral hemisphere. During development of postoperative neurological deficits, CBF was prominently increased in the left pre-motor area that the anastomosed STA perfused. In addition, CBF in the right cerebellar hemisphere was decreased when compared with CBF in the left cerebellar hemisphere. Central benzodiazepine receptor biding potential was prominently decreased in the left frontal lobe. On the 4 th postoperative month, increased CBF in the left pre-motor area and contralateral-to-ipsilateral cerebellar asymmetry of CBF resolved. The decrease in central benzodiazepine receptor biding potential in the left frontal lobe also resolved. During the patient's clinical course, electroencephalography was not performed.

\section{Discussion}

The present patient exhibited a prominent increase in CBF at the area that the anastomosed STA perfused during development of postoperative neurological deficits. Thus, this patient had cerebral hyperperfusion syndrome. Simultaneously, contralateral-to-ipsilateral cerebellar asymmetry of CBF was observed. This asymmetry may show crossed cerebellar hypoperfusion. Crossed cerebellar hypoperfusion is defined as a reduction in blood flow in the cerebellar hemisphere contralateral to a supratentorial lesion. ${ }^{11)}$ The mechanism underlying this phenomenon reportedly consists of disruption of the corticopontocerebellar pathway that causes functional deafferentation and transneuronal metabolic depression of the contralateral cerebellar hemisphere. ${ }^{12,13)}$ In patients with carotid artery occlusive disease, contralateral-to-ipsilateral cerebellar asymmetry of CBF reflects cerebral oxygen metabolism in the affected cerebral hemisphere relative to that in the contralateral cerebral hemisphere. ${ }^{14)}$ Thus, in the present patient, cerebral hyperperfusion may lead to reduction of cerebral metabolism, resulting in neurological deficits.

During development of postoperative neurological deficits, central benzodiazepine receptor biding potential was decreased in the area with hyperperfusion. These data suggested downregulation of cortical neurotransmitter receptor function due to cerebral hyperperfusion, also resulting in neurological deficits. Thus, findings among brain perfusion, cortical neurotransmitter receptor function, and symptoms are consistent each other.

After resolution of cerebral hyperperfusion syndrome, the contralateral-to-ipsilateral cerebellar asymmetry of $\mathrm{CBF}$ and the decrease in central benzodiazepine receptor biding potential in the affected hemisphere disappeared. These findings suggested that reduction of cerebral metabolism and downregulation of cortical neurotransmitter receptor function due to cerebral hyperperfusion is transient and reversible. The same findings including the symptoms were observed in a patient with nonconvulsive status epileptics, ${ }^{15)}$ suggesting that the present patient may have experienced such a status. However, the pathogenesis could not be determined because electroencephalography was not performed during her clinical course.

Receptors for other neurotransmitters such as dopamine and serotonin are distributed in the cerebral cortex, and their binding potentials are visualized using positron emission tomography. ${ }^{16,17)}$ In the present case, crossed cerebellar hypoperfusion that suggests a reduction in metabolism in the affected cerebral hemisphere was observed during cerebral hyperperfusion syndrome. Thus, other neurotransmitter receptors may also be downregulated in a cortical region with hyperperfusion. Our ${ }^{123} \mathrm{I}$-iomazenil SPECT findings may indicate downregulation of cortical neurotransmitter receptor function as a representative feature, although functions of other cortical neurotransmitter receptors were not investigated. Furthermore, how cerebral hyperperfusion reduces cerebral metabolism and downregulates neurotransmitter receptor function remains unresolved.

Using statistical imaging analysis for ${ }^{123}$ I-iomazenil SPECT, several investigators studied the relationship between cognitive function and central benzodiazepine receptor binding potential in the cerebral cortex in patients with adult MMD. ${ }^{18)}$ This study demonstrated that long-standing mild hemodynamic ischemia in the anterior circulation leads to a decrease in central benzodiazepine receptor binding potential within the medial frontal lobes, causing cognitive decline. ${ }^{18)}$ These findings suggested that ${ }^{123}$ I-iomazenil SPECT shows irreversible cortical neuron loss as well as reversible downregulation of cortical neurotransmitter receptor function. ${ }^{18)}$

\section{Conclusion}

Cerebral hyperperfusion after arterial bypass surgery for ischemic MMD may lead to transient, reversible reduction of cerebral metabolism and downregulation of cortical neurotransmitter receptor function, resulting in transient neurological deficits. 


\section{Conflicts of Interest Disclosure}

Kuniaki Ogasawara received a research grant from Nihon Medi-Physics Co., Ltd. Other authors do not have any conflicts of interest. All authors who are members of The Japan Neurosurgical Society (JNS) have registered online Self-reported COI disclosure statement forms through the website for JNS members. This work was partly supported by a Grants-in-Aid for Strategic Medical Science Research from the Ministry of Education, Culture, Sports, Science and Technology of Japan (S1491001); and for Scientific Research from the Japan Society for the Promotion of Science (JP18K09002).

\section{References}

1) Suzuki J, Takaku A: Cerebrovascular "moyamoya” disease. Disease showing abnormal net-like vessels in base of brain. Arch Neurol 20: 288-299, 1969

2) Research Committee on the Pathology and Treatment of Spontaneous Occlusion of the Circle of Willis; Health Labour Sciences Research Grant for Research on Measures for Infractable Diseases: Guidelines for diagnosis and treatment of moyamoya disease (spontaneous occlusion of the circle of Willis). Neurol Med Chir (Tokyo) 52: 245-266, 2012

3) Fujimura M, Tominaga T: Current status of revascularization surgery for moyamoya disease: special consideration for its 'internal carotid-external carotid (IC-EC) conversion' as the physiological reorganization system. Tohoku J Exp Med 236: 45-53, 2015

4) Fujimura M, Kaneta T, Mugikura S, Shimizu H, Tominaga T: Temporary neurologic deterioration due to cerebral hyperperfusion after superficial temporal artery-middle cerebral artery anastomosis in patients with adult-onset moyamoya disease. Surg Neurol 67: 273-282, 2007

5) Kim JE, Oh CW, Kwon OK, Park SQ, Kim SE, Kim YK: Transient hyperperfusion after superficial temporal artery/middle cerebral artery bypass surgery as a possible cause of postoperative transient neurological deterioration. Cerebrovasc Dis 25: 580-586, 2008

6) Ohue S, Kumon Y, Kohno K, Watanabe H, Iwata S, Ohnishi T: Postoperative temporary neurological deficits in adults with moyamoya disease. Surg Neurol 69: 281-286; discussion 286-287, 2008

7) Fujimura M, Mugikura S, Kaneta T, Shimizu H, Tominaga T: Incidence and risk factors for symptomatic cerebral hyperperfusion after superficial temporal artery-middle cerebral artery anastomosis in patients with moyamoya disease. Surg Neurol 71: 442-447, 2009

8) Fujimura $M$, Shimizu $H$, Inoue $T$, Mugikura $S$, Saito A, Tominaga T: Significance of focal cerebral hyperperfusion as a cause of transient neurologic deterioration after extracranial-intracranial bypass for moyamoya disease: comparative study with non-moyamoya patients using N-isopropyl-p-[(123)I] iodoamphetamine single-photon emission computed tomography. Neurosurgery 68: 957-964; discussion 964-965, 2011

9) Matsumoto Y, Ogasawara K, Saito H, et al.: Detection of misery perfusion in the cerebral hemisphere with chronic unilateral major cerebral artery stenoocclusive disease using crossed cerebellar hypoperfusion: comparison of brain SPECT and PET imaging. Eur J Nucl Med Mol Imaging 40: 1573-1581, 2013

10) Hatazawa J, Satoh T, Shimosegawa E, et al.: Evaluation of cerebral infarction with iodine 123-iomazenil SPECT. J Nucl Med 36: 2154-2161, 1995

11) Komaba Y, Mishina M, Utsumi K, Katayama $Y$, Kobayashi S, Mori O: Crossed cerebellar diaschisis in patients with cortical infarction: logistic regression analysis to control for confounding effects. Stroke 35: 472-476, 2004

12) Baron JC, Bousser MG, Comar D, Soussaline F, Castaigne P: "Crossed cerebellar diaschisis": a remote functional suppression secondary to supratentorial infarction in man. J Cereb Blood Flow Metab 1: s500, 1981

13) Pantano P, Baron JC, Samson Y, Bousser MG, Derouesne C, Comar D: Crossed cerebellar diaschisis. Further studies. Brain 109: (Pt 4) 677-694, 1986

14) Yamauchi H, Fukuyama H, Yamaguchi S, et al.: Crossed cerebellar hypoperfusion in unilateral major cerebral artery occlusive disorders. J Nucl Med 33: 1637-1641, 1992

15) Yoshida J, Komoribayashi N, Oikawa K, et al.: ${ }^{123}$ I-iomazenil single-photon emission computed tomography imaging in a patient with mild traumatic subdural hematoma accompanied with delayed transient aphasia. No Shinkei Geka in press (Japanese)

16) Caynas-Rojas S, Rodríguez-García G, Delint-Ramírez I, Miranda MI: Differential function of medial prefrontal cortex catecholaminergic receptors after long-term sugar consumption. Behav Brain Res 356: 495-503, 2019

17) Galusca B, Sigaud T, Costes N, Redoute J, Massoubre C, Estour B: Wide impairment of cerebral serotoninergic activity but inter-individual heterogeneity in bulimia nervosa patients: a pilot [(18)F]MPPF/PET study. World J Biol Psychiatry 15: 599-608, 2014

18) Nakagawara J, Osato T, Kamiyama K, et al.: Diagnostic imaging of higher brain dysfunction in patients with adult moyamoya disease using statistical imaging analysis for [123I]iomazenil single photon emission computed tomography. Neurol Med Chir (Tokyo) 52: 318-326, 2012

Address reprint requests to: Kuniaki Ogasawara, MD, Department of Neurosurgery, Iwate Medical University, 19-1 Uchimaru, Morioka, Iwate 020-8505, Japan. e-mail: kuogasa@iwate-med.ac.jp 